\section{Fürs Rauchen gibt es keine sichere Dosis}

Langjährige Raucher sterben früher auch dann, wenn ihr Konsum sich auf wenige Zigaretten am Tag beschränkt. Dies zeigt eine US-amerikanische Kohortenstudie mit 290.215 Teilnehmern, die zu ihrem Rauchverhalten befragt und sechs Jahre lang nachbeobachtet wurden. Von 19.857 aktiven Rauchern gaben $1.341(6,8 \%)$ bzw. $6.036(30,4 \%)$ an, aktuell weniger als eine bzw. ein bis zehn Zigaretten am Tag zu rauchen. Das Mortalitätsrisiko im Beobachtungszeitraum war in allen Rauchergruppen erhöht: Bei lebenslanger Beschränkung auf weniger als eine Zigarette am Tag lag es um 64\%, bei ein bis zehn Zigaretten um $87 \%$ höher als bei Nichtrauchern. Wenn in früheren Jahren mehr geraucht worden war, war das Risiko um den Faktor 2,1 bzw. 2,9 erhöht. Auch wer wenig raucht, hat also ein substanziell höheres Risiko für einen frühzeitigen Tod. „Es gibt kein risikofreies Niveau der Tabakrauchexposition", so die Autoren.

JAMA Intern Med, online 5. Dezember 2016; doi: 10.1001/jamainternmed.2016.7511

\section{Viel Alkohol erhöht das Schlaganfallrisiko}

Starker Alkoholkonsum erhöht das Risiko für einen Hirninfarkt, leichtes Trinken scheint einen protektiven Effekt zu haben. Dies zeigt eine Metaanalyse von 27 prospektiven Studien. 19.302 ischämische Schlaganfälle, 2.359 intrazerebrale und 1.164 Subarachnoidalblutungen wurden registriert. Der Alkoholkonsum wurde mittels Fragebögen ermittelt, dabei ging man von $12 \mathrm{~g}$ Alkohol pro Drink aus. Das relative Risiko (RR) für einen ischämischen Infarkt war bei weniger als einem Drink pro Tag um 10\%, bei ein bis zwei Drinks um $8 \%$ reduziert. Ein Alkoholkonsum von zwei bis vier Drinks erhöhte dagegen das Risiko um 8\%, ein erheblicher Konsum von mehr als vier Drinks pro Tag sogar um 14\%. Auf das Risiko für hämorrhagische Schlaganfälle hatte leichter Alkoholgenuss keinen Einfluss. Mehr als vier Drinks pro Tag steigerten hingegen auch hier das Risiko signifikant.

BMC Med. 2016;14:178

\title{
Joggen macht Spermien mobil
}

Körperliche Aktivität nimmt Einfluss auf immunologische und oxidative Vorgänge in Körperflüssigkeiten, Organen und Geweben. Doch nur wenige Daten existieren bislang dazu, welche Auswirkungen ein kontinuierliches Training auf die Fortpflanzungsfähigkeit hat. Nun haben Reproduktionsexperten um Behzad Hajizadeh Maleki von der Universität Gießen in einer randomisierten, kontrollierten Studie den Effekt von Sport unterschiedlicher Intensität auf männliche Fruchtbarkeitsmarker analysiert.

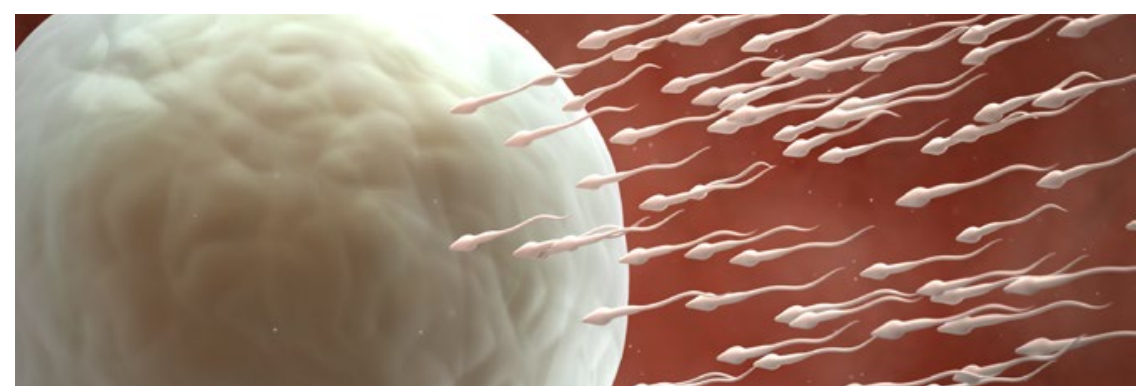

$\mathrm{D}$ abei wurden 280 gesunde Männer zwischen 25 und 40 Jahren nach dem Zufallsprinzip auf eines von drei Trainingsmodulen (kontinuierliches Training moderater Intensität, MICT; kontinuierliches Training hoher Intensität, HICT; Intervalltraining hoher Intensität, HIIT) und eine Nichtsportgruppe verteilt. $\mathrm{Zu}$ Studienbeginn, nach 12 und 24 Wochen Training sowie 7 und 30 Tage nach Trainingsende wurden im Sperma Entzündungsmarker und Marker für oxidativen Stress bestimmt. Zusätzlich wurden die Samenzellen auf Qualität, Intaktheit und DNA-Schäden untersucht. Nach 24 Wochen kontinuierlichen Trainings hatten sich die Indikatoren für oxidativen Stress und Inflammation bei den gesunden Männern aller drei Sportgruppen gegenüber den Ausgangswerten signifikant verringert. Die Vorteile zeigten sich noch sieben, I zum Teil auch noch 30 Tage nach Beenहुํํㄹ digung des Trainings. Diese Verände密 rungen wiederum wirkten sich positiv auf die Samenqualität und die Unversehrtheit der DNA aus.

Als günstigste Trainingsform für die männliche Reproduktionsfähigkeit ermittelten Maleki und Kollegen die MICT. Hinsichtlich Beweglichkeit, Morphologie und Anzahl waren die Spermien der Probanden, die moderat trainiert hatten, denen der anderen Gruppen nach 12 und 24 Wochen überlegen. In der MICT-Gruppe waren die Männer in den ersten zwölf Wochen an drei bis vier Tagen pro Woche jeweils 25-30 Minuten auf dem Laufband gejoggt oder gelaufen. Danach wurde das aerobe Training auf jeweils 40-45 Minuten an vier bis sechs Tagen pro Woche gesteigert.

Die Studienautoren gehen davon aus, dass auch trainingsbedingte Veränderungen bei Gewicht, BMI, Körperfett und Taillenumfang zu den antiinflammatorischen Effekten beigetragen haben. In diesen Bereichen erzielten alle trainierenden Männer im Vergleich zur Nichttrainingsgruppe nach 24 Wochen signifikante Vorteile.

In dieser Studie, so Maleki und Kollegen, habe sich gezeigt, dass Intensität, Dauer und Art des Sportprogramms berücksichtigt werden müssten, wenn der Einfluss körperlicher Aktivität auf die Reproduktionsfähigkeit untersucht werde. Wie sich die Veränderungen der Marker letztlich auf die Reproduktionsfähigkeit eines Mannes auswirkten, bleibe abzuwarten. Vielleicht können Männer mit Kinderwunsch in absehbarer Zeit entsprechende Trainingsempfehlungen berücksichtigen, um ihrer Familienplanung schneller Hand und Fuß zu verleihen.

Reproduction February 1, 2017, 153: 157-174; doi:10.1530/REP-16-0318 